\title{
KOALA, a wide-field 1000 element integral-field unit for the Anglo- Australian Telescope: assembly and commissioning
}

\author{
Ross Zhelem*a, Jurek Brzeski ${ }^{\mathrm{a}}$, Scott Case $^{\mathrm{a}}$, Vlad Churilov ${ }^{\mathrm{a}}$, Simon Ellis ${ }^{\mathrm{a}}$, Tony Farrell ${ }^{\mathrm{a}}$, Andrew \\ Green $^{\mathrm{a}}$, Anthony Heng ${ }^{\mathrm{a}}$, Anthony Horton ${ }^{\mathrm{a}}$, Michael Ireland ${ }^{\mathrm{b}}$, Damien Jones ${ }^{\mathrm{c}}$, Urs Klauser ${ }^{\mathrm{a}}$, Jon \\ Lawrence $^{\mathrm{a}}$, Stan Miziarski ${ }^{\mathrm{a}}$, David Orr ${ }^{\mathrm{a}}$, Naveen Pai ${ }^{\mathrm{a}}$, Nick Staszak ${ }^{\mathrm{a}}$, Julia Tims ${ }^{\mathrm{a}}$, Minh Vuong ${ }^{\mathrm{a}}$, Lew \\ Waller $^{\mathrm{a}}$ and Pascal Xavier ${ }^{\mathrm{a}}$ \\ a Australian Astronomical Obseratory, 105 Delhi Rd, North Ryde NSW 2113, Australia; \\ ${ }^{b}$ Research School of Astronomy and Astrophysics, Mount Stromlo Observatory, Cotter Rd., Weston \\ Creek ACT 2611, Australia; \\ ${ }^{\mathrm{c}}$ Prime Optics, 17 Crescent Rd., Eumundi QLD 4562, Australia
}

\begin{abstract}
The KOALA optical fibre feed for the AAOmega spectrograph has been commissioned at the Anglo-Australian Telescope. The instrument samples the reimaged telescope focal plane at two scales: $1.23 \mathrm{arcsec}$ and 0.70 arcsec per image slicing hexagonal lenslet over a 49x27 and 28x15 arcsec field of view respectively. The integral field unit consists of 2D hexagonal and circular lenslet arrays coupling light into 1000 fibres with 100 micron core diameter. The fibre run is over $35 \mathrm{~m}$ long connecting the telescope Cassegrain focus with the bench mounted spectrograph room where all fibres are reformatted into a one-dimensional slit. Design and assembly of the KOALA components, engineering challenges encountered, and commissioning results are discussed.
\end{abstract}

Keywords: integral field unit, optical fibres, image slicer, microlens array, spectroscopy

\section{INTRODUCTION}

Integral field spectroscopy is an efficient method to study extended spatially resolved objects. The telescope image of a seeing limited point source or a $2 \mathrm{D}$ object is sampled by an image segmenter to the required angular dimension on the sky, then each sample is analysed spectrally. There are many methods to perform 2D sampling. The Australian Astronomical Observatory has built integral field units (IFU) that are based on the use of optical fibres.

Fibre based instruments have some significant advantages over optical IFUs that are integrated into a spectrograph. Fibre feeds can deliver beams to spectrographs that are located in a thermally and vibrationally controlled environment for better stability during observations and over the length of a survey. They are also very compact, lightweight and rigid. However, miniature optics require high precision assembly. This paper describes the design and integration of KOALA concentrating on the assembly of its micro-optics.

KOALA is an upgrade to SPIRAL which had had two phases of development. SPIRAL A was a prototype instrument with 37 spatially resolved elements. A larger array IFU $^{2}$ was built for SPIRAL B covering a 22" x 11" field. It consisted of 512 microlenses (each $1 \mathrm{~mm}$ square), providing a spatial resolution of 0.7 " per lens. Originally, SPIRAL B fed its own bench mounted spectrograph. After commissioning AAOmega ${ }^{3}$, SPIRAL B was retrofitted to deliver input for the new spectrograph.

*ross.zhelem@aao.gov.au; phone 612 9372-4872; fax 612 9372-4880; aao.gov.au 
Integral field spectroscopy with SPIRAL B and AAOmega became popular but has been limited by the optical throughput of the fibres and the size of the array. KOALA which will feed the AAOmega spectrograph, is designed to provide higher throughput, a larger field of view, and greater flexibility than SPIRAL. The KOALA IFU samples telescope image at 0.7 " or 1.23 " per lens using interchangeable fore-optics. The image segmenter of KOALA uses a hexagonal microlens array with elements $0.25 \mathrm{~mm}$ across (flat to flat) produced on a single substrate. It contains 1000 microlenses covering the field of view 28 " x 15 " or 49 " x 27 " depending on the magnification used.

\section{KOALA OPTICS}

\subsection{Fold mirror}

The KOALA fibre feed is schematically shown in Fig. 1. It consists of a fold mirror, fore-optics magnifiers, an IFU with microlens arrays, a fibre run to the spectrograph room and the slit unit feeding the AAOmega spectrograph.

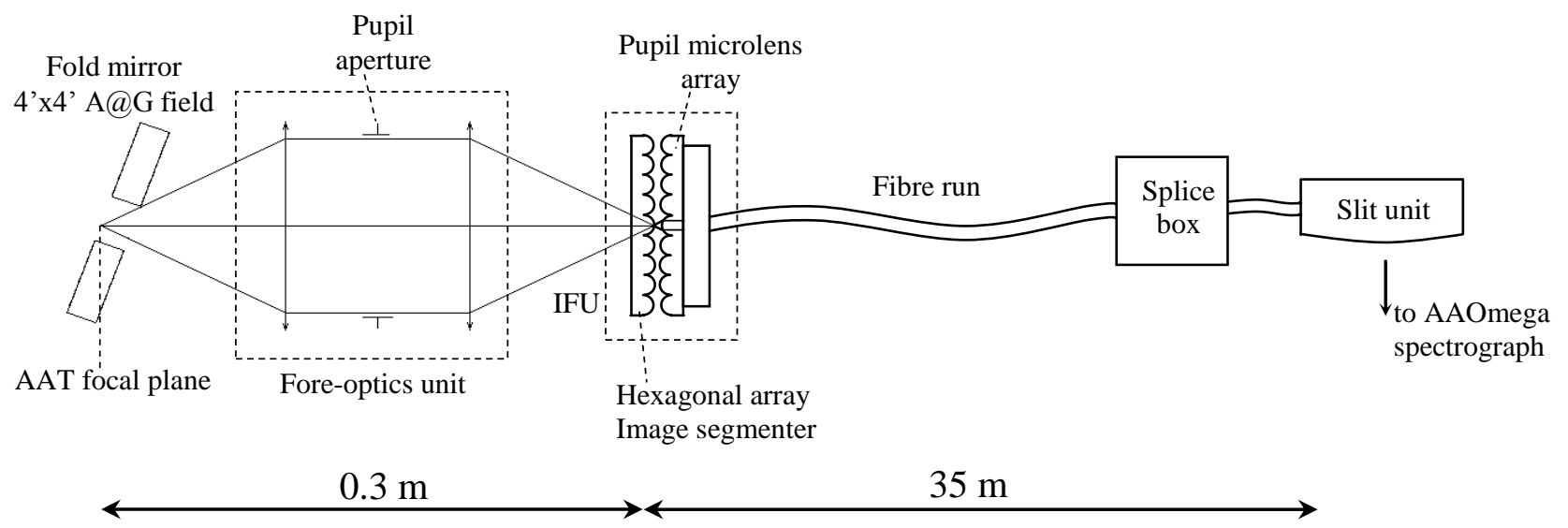

Figure 1. Schematic showing the main elements of the KOALA fibre feed.

The fold mirror is located in the focal plane of the Anglo-Australian Telescope (AAT). The science beam passes through a central aperture in the fold mirror and the outer $4 \times 4$ arc minute field is reflected to the acquisition and guiding system of the dedicated Cassegrain focal station CURE (Cassegrain Unit Refurbished Environment). KOALA is the second instrument built for CURE that is meant to become the standard facility for fibre instruments at the Cassegrain focus ${ }^{1}$. Acquisition and guiding are provided with the help of a CCD camera viewing the telescope focal plane de-magnified by the factor of two. Additionally, the station has its own calibration facility with a quartz tungsten halogen lamp for flat fielding and ThAr, CuAr and FeAr hollow cathode lamps for wavelength calibration. The hardware of the calibration system was upgraded to accommodate the larger field of view of KOALA.

\subsection{Fore-optics}

The purpose of the fore-optics unit is to provide the telecentric input on the IFU at an optimum scale. The first element of the fore-optics assembly is a magnifying triplet lens, the second element is a doublet field lens providing telecentricity ${ }^{4}$. All lenses are made of OHARA i-line glasses and BBAR coated to maximize throughput for the wavelength range $370-950 \mathrm{~nm}$. There are two sets of fore-optics delivering 1.23 " and 0.7 " per IFU lenslet element with magnification $1.35 \mathrm{x}$ and $2.35 \mathrm{x}$ respectively.

Both fore-optics magnifiers are integrated with a fold mirror with the aperture of appropriate size (Fig. 2). The choice of fore-optics can be made from the instrument user interface. The fore-optics lenses were designed with the mechanical constraint of $300 \mathrm{~mm}$ distance between the telescope focal plane and the IFU. The lens parameters were chosen based on the size of the field and the telecentricity requirement. The fore-optics lenses deliver less than $20 \mu \mathrm{m}$ RMS radius image quality across the entire field of view, the cross talk between adjacent IFU lenses becomes insignificant. 

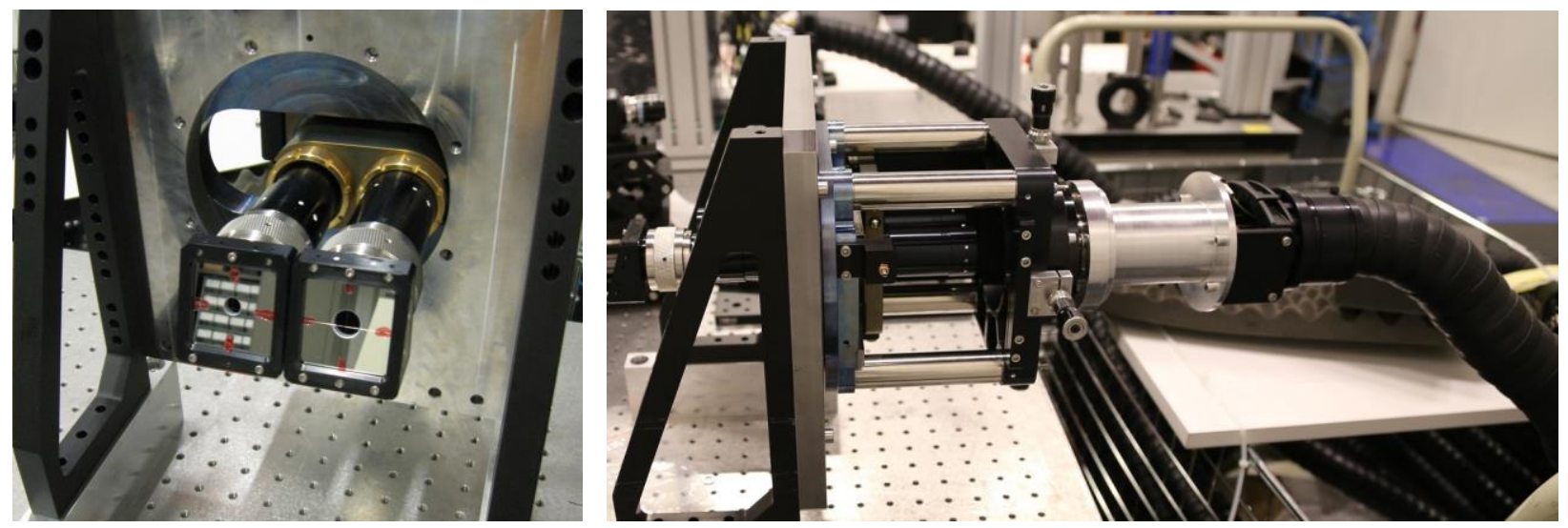

Figure 2. KOALA assembly in the laboratory. Temporary alignment cross hair can be seen over the fold mirrors. 0.7" scale fore-optics unit is deployed by the interchange mechanism. The fore-optics is followed by the IFU housing and the fibre cable run.

\subsection{Microlens arrays}

Microlens arrays split the magnified telescope image into individual elements and form the image of the telecentric pupil on a fibre core. In principle, a single lens (array) can perform this function. However, the beam will be affected by the geometric focal ratio degradation (FRD) imposing additional constraints ${ }^{4}$, hence, the design with two microlenses was selected for the KOALA IFU (Fig. 3). The fibre input is collimated with a beam focal ratio of F3.2 for both image scales. The AAOmega spectrograph accepts F3.1 input with some margin for alignment and fibre FRD.
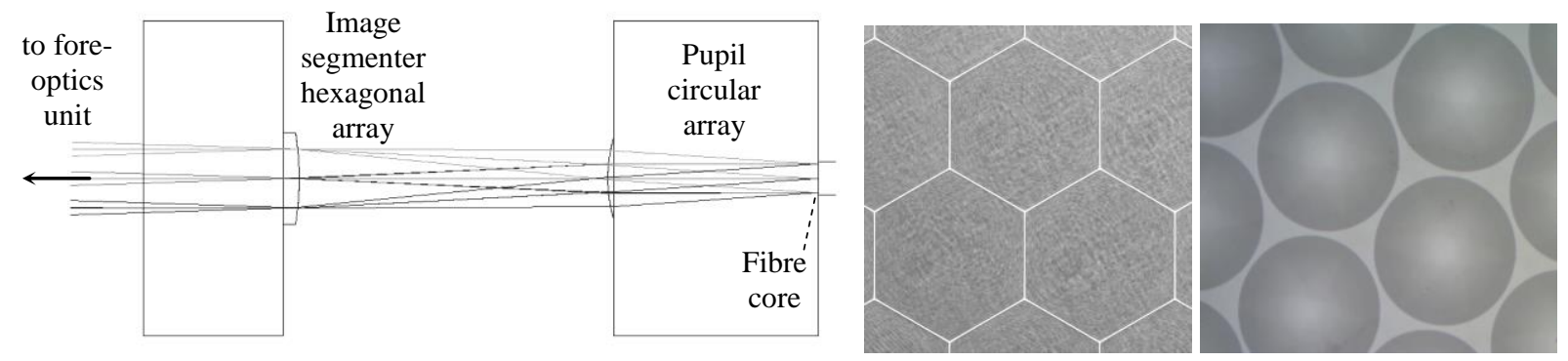

Figure 3. KOALA IFU microlens layout. Photographs of hexagonal and circular microlens arrays.

The KOALA microlens arrays were subjected to thorough metrology verification ${ }^{5}$. The first microlens array is the image segmenter. It is important that the optical apertures of all lenslets are continuous with minimum edge gap. Light falling into the gap will contribute to loss. Additionally, the lenslet surface figure must conform to the requirements of image quality because it is involved into the formation of the pupil image. Hexagonal lenslets of the image segmenter array have precise geometry based on $250 \mu \mathrm{m}$ pitch flat to flat, they are separated by $\sim 5 \mu \mathrm{m}$ gap. Throughput measurements confirmed that the fill factor of the array is $94 \%$ excluding Fresnel losses. The KOALA hexagonal array was successfully produced by Jenoptik Optical Systems by means of plasma etching in a fused silica substrate with the average surface figure error less than $40 \mathrm{~nm}$ RMS.

The second (pupil) microlens array consists of lenslets of circular aperture. They are hexagonally packed to match the image segmenter array. Surface figure is under $50 \mathrm{~nm}$ RMS. This is an off the shelf product of SUSS MicroOptics. Originally $1 \mathrm{~mm}$ thick, the array was thinned to $0.7 \mathrm{~mm}$ according to the optical prescription. The pupil microlens array was delivered to the AAO bonded to the fibre array by the IFU manufacturer Fiberguide Industries.

Both arrays are diced to a rectangle $10 \times 15 \mathrm{~mm} .1000$ active microlenses/fibres are configured with the aspect ratio 2 : 1.1 that is suitable for extended elongated objects. The microlens arrays are separated by $0.91 \mathrm{~mm}$ between lens vertices, lens surface radii of curvature are $0.88 \mathrm{~mm}$ and $0.45 \mathrm{~mm}$ for image segmenter and the pupil arrays respectively. The beam footprint on the pupil microlens is $230 \mu \mathrm{m}$ for 1.23 " scale and $194 \mu \mathrm{m}$ for 0.7 " scale. The diameter of the pupil lens is $244 \mu \mathrm{m}$. Close match calls for tight requirements on the alignment of both arrays. In order to ensure the 
performance at the larger scale, a $3 \mu \mathrm{m}$ tolerance was achieved for image segmenter array positioning with respect to the pupil array.
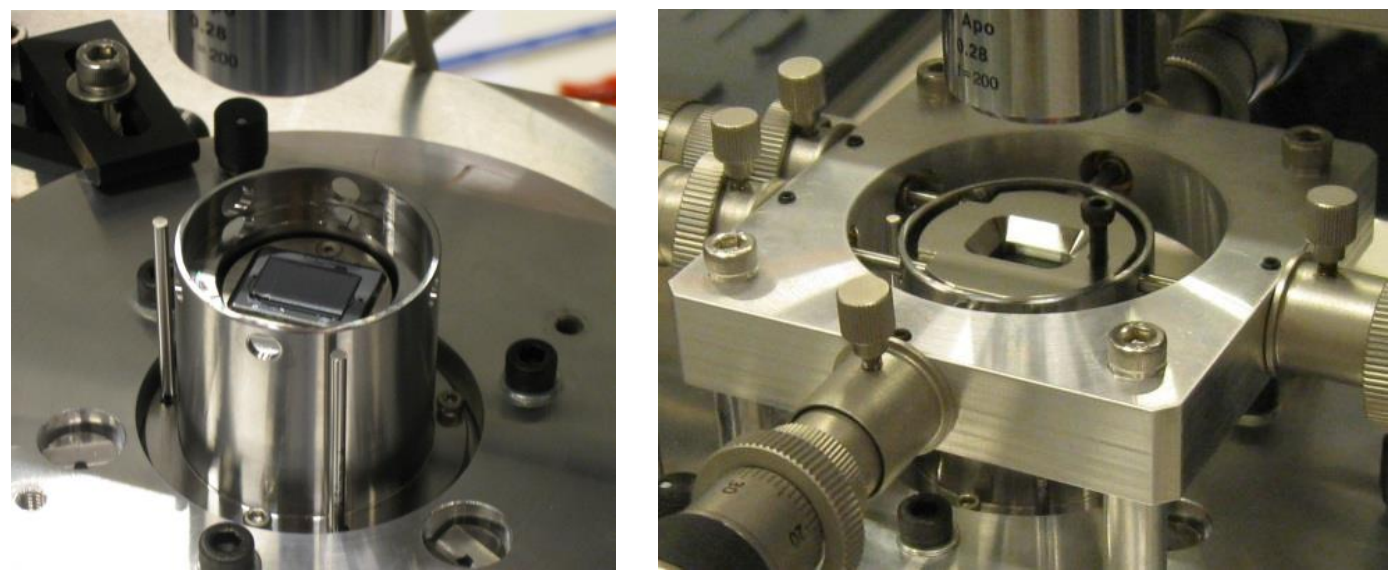

Figure 4. Pupil microlens array bonded to the IFU (left). Assembly of the hexagonal microlens array (right)

The KOALA fibre array is assembled using Ceramoptec Optran WF 100/125/250 fibre. The fibre core is $100 \mu \mathrm{m}$ in diameter, cladding $125 \mu \mathrm{m}$ and optional coating $250 \mu \mathrm{m}$. The double microlens array forms the image of the telecentric pupil on each fibre. The diameter of the pupil image is $92 \mu \mathrm{m}$ for 1.23 " scale and $53 \mu \mathrm{m}$ for 0.7 " scale. Clearance from the edge will help to accommodate blurring due to aberration and diffraction. The pupil is well undersized for the smaller scale but more challenging for the 1.23" scale fore-optics. It is obvious that the error in pupil footprint on a fibre core may be compensated by the tilt of the IFU with respect to the fore-optics. However, in the double microlens array configuration, such a tilt may cause vignetting at the pupil microlenses.

\subsection{Alignment strategy}

The alignment setup for microlens arrays is based on the precision 3 axis stage. The stage carries a point source microscope tool ${ }^{6}$ looking down on the microlens arrays. The hexagonal microlens housing is shown in Fig. 4. The microlens array is bonded to the cell, then centred optically with respect to the pupil array and fixed to the housing using RTV. The point source microscope enables direct viewing of a fibre core through a pupil microlens as well as the referencing of fiducial points of microlenses in both arrays. Separation between arrays is controlled simultaneously with lateral positioning, it is set to the optimised prescription value to within $10 \mu \mathrm{m}$.

The instrument assembly was accompanied by alignment and testing. The fold mirror, fore-optics and the IFU were mounted on a common optical axis. The fold mirror and the IFU are optically conjugated so that when an object is in focus on the acquisition and guiding camera, its image is focused on the image segmenter array (Fig. 5, left panel). The axial position of the fold mirror was also set with respect to the KOALA mounting interface.
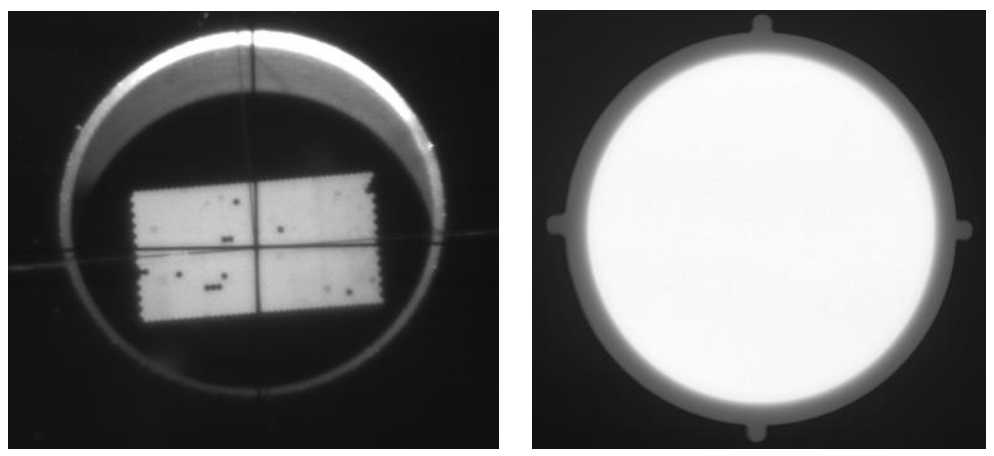

Figure 5. The image of the IFU built by the fore-optics unit (left). IFU tilt alignment with respect to the fore-optics pupil aperture fiducials (right).

At the telescope, the location of the KOALA fold mirror was verified on the acquisition and guiding camera and the IFU tilt was finalised to image the telescope pupil onto a fibre core. To achieve pupil alignment, the IFU was back 
illuminated through the slit and fibre run (Fig. 1) and the magnified image of the fibre was observed at the fore-optics pupil aperture with the help of a pupil viewing camera (Fig. 5, right). The IFU was then detached from the fore-optics and the telescope pupil viewed through the magnifier lens. Both pupils were made concentric with respect to the pupil aperture fiducials.

\subsection{IFU and fibre run}

The KOALA IFU consists of 1000 microlenses/fibres covering a rectangular imaging area (Fig. 6). The fibre cable run is $35 \mathrm{~m}$ long, it connects the IFU in the AAT Cassegrain cage with the slit in the AAOmega spectrograph room. The 2D imaging field is reformatted into a linear slit feeding the spectrograph. The IFU and slitlets are built as separate units. The fibres from the IFU and the slit join at the splice box. Each fibre is fusion spliced and the join is connected with a splice sleeve.

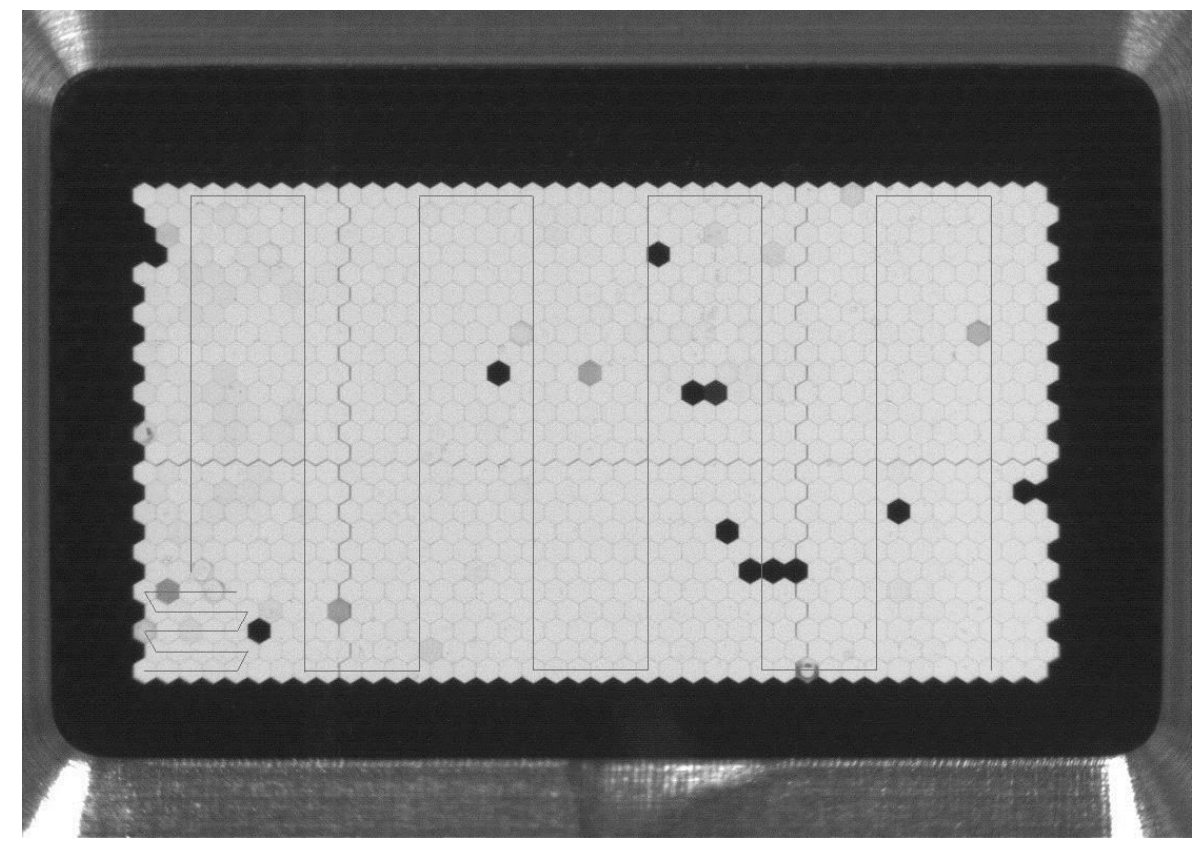

Figure 6. KOALA IFU back illuminated through the slit. The line follows the path of increasing numbers of the IFU elements reformatted into the linear slit shown in figure 7.

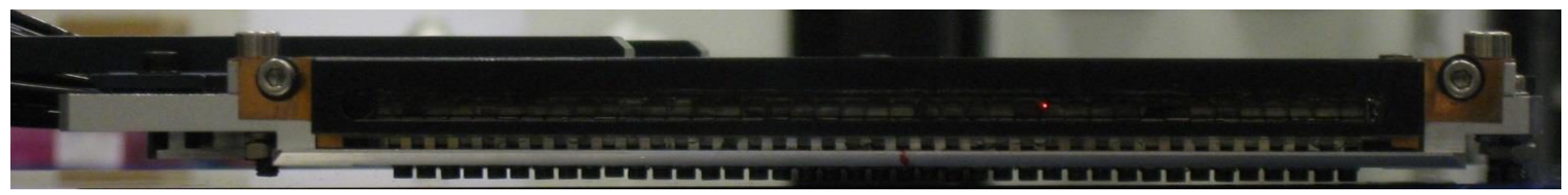

Figure 7. KOALA slit for AAOmega spectrograph with a single fibre illuminated through the IFU. Slitlets are fixed in the housing and optically greased to the curved field lens.

The SPIRAL IFU had very low throughput at $950 \mathrm{~nm}$ caused by 'wet' fibres. The Ceramoptec water free (WF) fibres used for KOALA offer significant improvement across the entire visible wavelength range. The IFU fibres have an outside diameter of $250 \mu \mathrm{m}$ determined by the coating. They are assembled in bundles of 25 fibres contained in a single pvc tube. The tube runs along with a Kevlar strain relief fibre inside of an additional outer tube forming a bundle. There are 40 bundles, each terminating in an individual slitlet. The numbering of fibres in the IFU is shown in Fig. 6 with detailed path of increasing numbers for the first bundle. The pattern is spread across the entire IFU. Fibre packaging and numbering targets the efficient reformatting of the imaging area into a pseudo-slit by means of which close lenslets on the IFU form adjacent spectra of comparable intensity. Few low transmission fibres can be easily identified as a result of the IFU and fibre cable fabrication. Overall operability of the IFU elements is greater than the requirement of $98 \%$. 


\subsection{Slit unit}

The AAOmega spectrograph slit (Fig. 7) has an active length of $145 \mathrm{~mm}$ along an arc of $536 \mathrm{~mm}$ radius. Positioning 1000 KOALA fibres presented some challenges for the slit fabrication as compared to the AAOmega native slit (with 392 fibres) and the SPIRAL-B fibre feed (512 fibres). The slitlet parameters were carefully chosen to ensure that defocus of the centre of a slitlet with respect to the edge as well as the cross talk of adjacent spectra are negligible. Each individual slitlet contains 25 fibres from a single bundle at $133 \mu \mathrm{m}$ core to core spacing with a total length of $3.45 \mathrm{~mm}$. The slitlets are composed of a fused silica v-groove with lid. The fibre coating is stripped prior to insertion. Routing large amount of fibres with coating within a limited space resulted in some focal ratio degradation. The slit housing features a low profile design to minimize the obstruction of the beam reflected from the spectrograph collimator mirror.

\section{MECHANICAL LAYOUT}

The KOALA mechanical layout allows the instrument to interface with the existing CURE Cassegrain focal station. The structure allows the two sets of fore-optics units to be interchanged remotely. The structure of the KOALA instrument also allows for kinematic mounting of the IFU and its removal for maintenance purposes if required. While not in use the instrument is stored in the Cassegrain cage of the telescope.
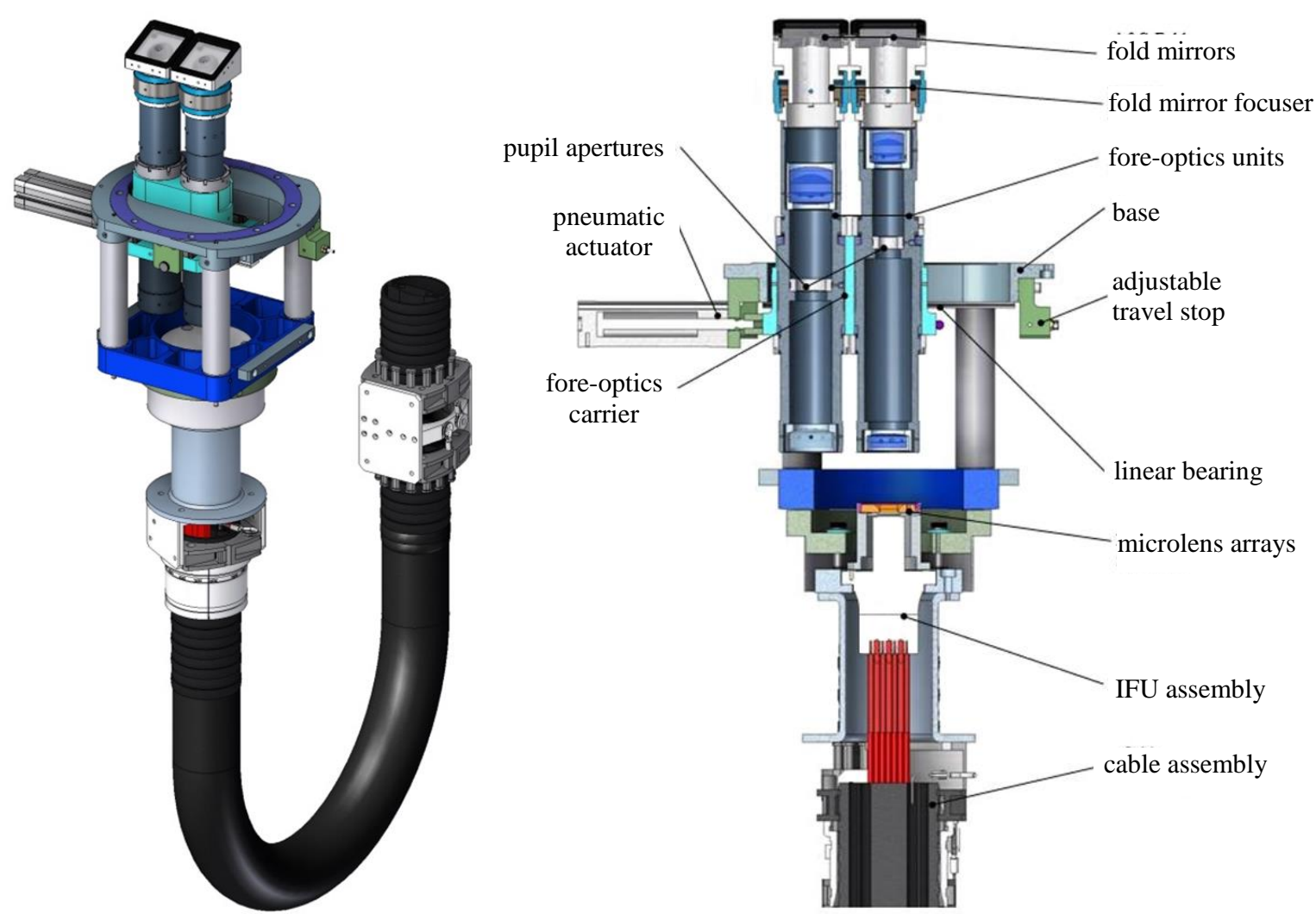

Figure 8. Isometric and plane views of the KOALA fore-optics unit interfaced with the IFU.

The base of the KOALA fore-optics structure is manufactured of stainless steel. It provides the interface to CURE and the foundation to mount the Thomson linear bearings that allow the fore-optics to exchange. The fore-optics exchange is actuated through a FESTO pneumatic actuator. Two adjustable hard stops at the end of travel give positional stops. The mechanism provides for the repeatability of the fore-optics positioning to within $30 \mu \mathrm{m}$. Due to space constraints the tiptilt of each fore-optics unit was held, successfully, with manufacturing tolerances. The KOALA instrument only bolts to the CURE interface. After final xy alignment to the secondary mirror was achieved, two holes were transfer drilled into CURE to allow repeatable positioning when KOALA is removed from CURE. 
The fore-optics barrels were machined out of aluminum. Lenses were mounted in aluminum subcells to allow precision tip-tilt adjustment at the cell level and xy alignment at the barrel level. The barrels' design included a precision diameter that slip fit into the instrument carrier. Two opposing threads retain the barrel to the carrier and allow Z-axis adjustment. Each fore-optics also contains a fold mirror that is mounted on a focuser to allow each unit to be confocal with the acquisition and guiding camera when in the observing position. The IFU cable assembly is mounted kinematically to the KOALA structure. The kinematic mount has an integral focus and tip-tilt adjustment which allows for final alignment of the IFU to the fore-optics.

\section{ELECTRONICS AND SOFTWARE}

The KOALA instrument observing system software integrates control of the KOALA instrument lamp and FOV controller with control of the AAOmega Spectrograph, CCD systems and telescope. Scripted or on-demand observing is available, as is automated spectrograph focus via two different techniques. This was accomplished via efficient integration of existing software to become the KOALA control system. The starting point was the older SPRIAL instrument ${ }^{2}$ observing system software. This integrated the AAO's older IFU with the AAOmega spectrograph. The SPIRAL software was itself an adaption of the $2 \mathrm{dF} / \mathrm{AAOmega}$ observing system ${ }^{7}$, which has proved to be an easily adaptable and highly flexible system.

A new KOALA mode was added to the control task, similar in many ways to the existing SPIRAL mode. Differences from SPIRAL included generating a FITS fibre table appropriate for the larger IFU, operation of the CURE lamp controller in place of the older AAO SPIRAL Cassegrain lamp system and the addition of the FOV selector. The CURE lamp controller had been commissioned as part of the CYCLOPS2 instrument ${ }^{1}$. For KOALA, additional lamps were to be added as well as the FOV selector. This required a rewrite of the controller firmware and related components of the higher-level CURE software. The high-level interface to CURE for CYCLOPS2 did not match the interface used by the older lamp systems used by SPIRAL and $2 \mathrm{dF}$. To avoid significant changes to the complex Control Task, an additional commands and monitoring points were added to the CURE so as to match the SPIRAL interface.

\section{KOALA THROUGHPUT}

The KOALA throughput was measured in the laboratory using a $635 \mathrm{~nm}$ laser source. The beam focal ratio was set to F/7.9 to simulate the telescope input, and a focused spot was projected onto a single microlens through the fore-optics. The throughput value is the ratio of transmitted and input power measured by a laser power meter. In this way, the instrument throughput was measured for all the system shown in Fig. 1. However, due to the limited coverage of a microlens with the test beam, the measured throughput value must be scaled by the fill factor of the hexagonal segmenter array.

The throughput of individual components of the instrument was also characterized. The fore-optics magnifier and hexagonal array (including fill factor) were measured separately. Prior to the microlens assembly, measurements were taken for the fibre cable with the pupil microlens array bonded to the IFU and terminated at the slit with the slit lens on the opposite end. Both approaches yield comparable values (Table 1).

Table 1. KOALA throughput measurements in the laboratory.

\begin{tabular}{r|c} 
Element & Throughput \\
\hline point source input & 0.83 \\
hex array fill factor & 0.94 \\
\hline total & $0.78 \pm 0.02$
\end{tabular}

\begin{tabular}{r|c} 
Element & Throughput \\
\hline fore-optics unit & 0.94 \\
hex array & 0.91 \\
pupil array+fibre cable+slit lens & 0.90 \\
\hline total & $0.77 \pm 0.03$
\end{tabular}

The blue performance of the instrument is determined by the properties of fibres and the internal transmission of the fore-optics unit. The plot of KOALA transmission in the lab in Fig. 9 is produced using the measured red performance as the reference point. 


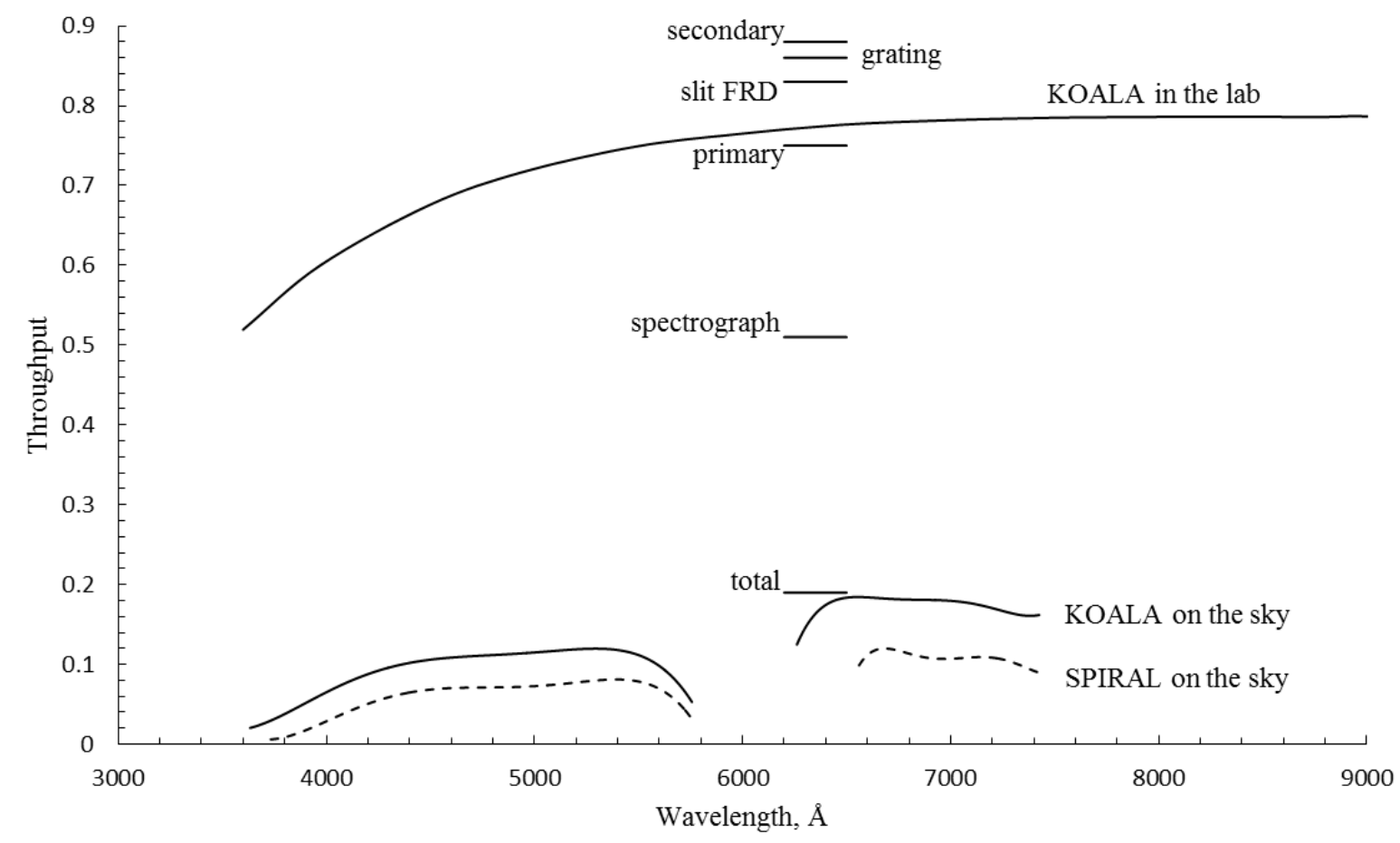

Figure 9. KOALA throughput measurements in the laboratory and on the sky with contributions from the AAT mirrors and the AAOmega spectrograph.

The full spectrum characterization was done on the sky taking multiple spectra of spectrophotometric standard stars produced by the AAOmega spectrograph fed by KOALA or SPIRAL-B. The comparative average result is shown in Fig.9 for KOALA and SPIRAL-B fibre feeds. The same 580V and 1000R diffraction gratings were used in both measurements, the effects of atmospheric extinction and the telescope central obscuration are taken out during data processing. According to Fig. 10 KOALA delivers more than 1.5 the throughput of SPIRAL. The blue throughput is significantly higher due to the use of the selected fibres.

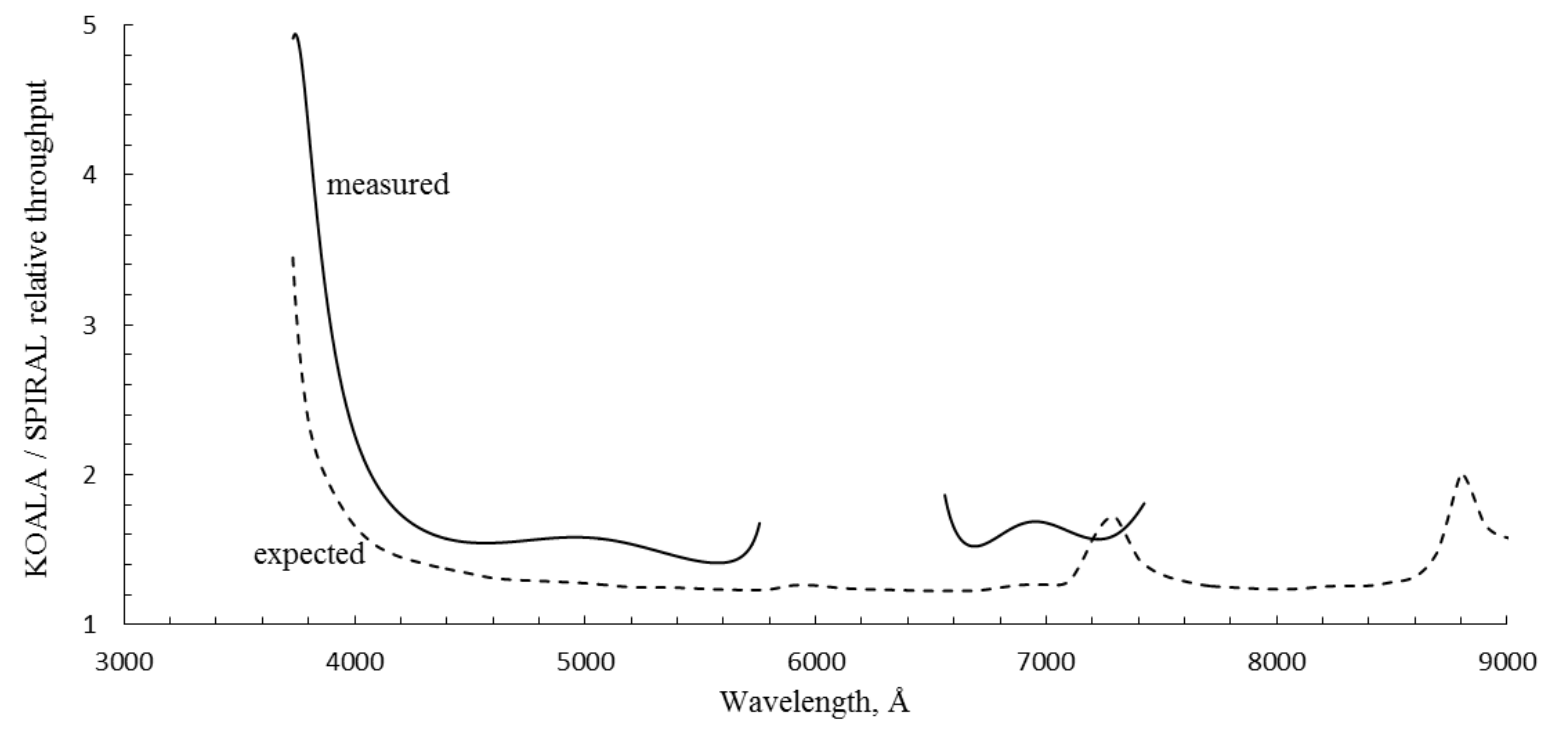

Figure 10. Expected and measured relative efficiency of KOALA vs SPIRAL. 
Contributions into the throughput from the AAT mirror coatings and the AAOmega spectrograph are based on measured values. The reflectivity of the AAT mirror was measured prior to the last aluminizing run. FRD caused by the slit is the average value (>0.75 requirement) that accounts for the vignetting loss at the spectrograph stop. The AAOmega spectrograph throughput was determined during the instrument commissioning. The convolution of telescope, the KOALA fibre feed and the AAOmega spectrograph at $635 \mathrm{~nm}$ is in good agreement with the end-to-end direct measurements. The lower overall transmission in the blue wavelength range is the property of the AAOmega blue camera CCD that has been upgraded shortly after KOALA commissioning.

\section{SUMMARY}

KOALA is intended to provide the Anglo Australian Telescope with an improved wide field IFU capability for the AAOmega spectrograph. The instrument capitalizes on the technological advancement in high throughput optical fibres and precision microlens technology. It is expected to be a powerful instrument for obtaining information on extended astrophysical sources such as star clusters, planetary nebulae, and nearby galaxies.

\section{REFERENCES}

[1] Horton, A., Tinney, C. G., Case, S., Farrell, T., Gers, L., Jones, D., Lawrence, J.,Miziarski, J., Staszak, N., Orr, D., Vuong, M., Waller, L. and Zhelem, R., "CYCLOPS2: the fibre image slicer upgrade for the UCLES high resolution spectrograph," Proc. SPIE 8446, 84463A (2012)

[2] Lee, D. and Taylor, K., "Fiber developments at the Anglo-Australian observatory for SPIRAL and AUSTRALIS," Proc. SPIE 4008, 268 (2000)

[3] Sharp, R., Saunders, W., Smith, G., Churilov, V., Correll, D., Dawson, J., Farrel, T., Frost, G., Haynes, R., Heald, R., Lankshear, A., Mayfield, D., Waller, L. and Whittard, D., "Performance of AAOmega: the AAT multi-purpose fiber-fed spectrograph," Proc. SPIE 6269, 62690G (2006)

[4] Ellis, S. C., Ireland, M., Lawrence, J. S., Tims, J., Staszak, N., Brzeski, J., Parker, Q. A., Sharp, R., BlandHawthorn, J., Case, S., Colless, M., Croom, S., Couch, W., De Marco, O., Glazebrook, K., Saunders, W., Webster, R. and Zucker, D. B., "KOALA: a wide-field 1000 element integral-field unit for the AngloAustralian Telescope," Proc. SPIE 8446, 84460V (2012)

[5] Lee, D. and Haynes, R., "Characterization of lenslet arrays for astronomical spectroscopy," PASP 113, 1406$1419,(2001)$

[6] Parks, R. E., "Lens centering using the point source microscope," Proc. SPIE 6676, 667603 (2007)

[7] Smith, G. A., Saunders, W., Bridges, T., Churilov, V., Lankshear, A., Dawson, J., Correll, D., Waller, L., Haynes, R., Frost, G., "AAOmega: a multipurpose fiber-fed spectrograph for the AAT," Proc. SPIE 5492, 410420 (2004) 